\title{
EDITORIAL
}

\section{The "ultrasonic stethoscope": is it of clinical value?}

\section{A Salustri, P Trambaiolo}

The availability of miniaturised ultrasound instruments, such as the "ultrasonic stethoscope", herald a new era in the detection of important cardiovascular pathology at the point-of-care

See end of article for authors' affiliations

......................

Correspondence to: Dr Alessandro Salustri, Cardiology Unit, POI Portuense, Via dei Buonvisi, 50-00148 Roma, Italy; salustri@jnet.it
$\mathrm{D}$ uring the last centuries, cardiologists were taught to perform physical examination by using their senses; indeed, most clinical diagnoses are still based on auscultation which requires the best skill to recognise abnormal sounds and different types of heart murmurs. However, awareness that abnormal physical findings are not always specific nor always sensitive has led to the development of an armamentarium of diagnostic procedures during the last few decades. In particular, ultrasound imaging allows the cardiac structures to be viewed dynamically, undoubtedly providing a new window on the heart. Currently, echocardiography is the most widely used and cost effective diagnostic imaging tool in cardiology and has largely replaced other imaging modalities in a wide variety of health care environments. Generally, a standard echocardiogram is requested whenever the physical examination is inconclusive or doubtful, or for evaluation of the severity of a known disease.

However, echocardiography is becoming more and more complex and the significant equipment costs, standardised examinations, and required specialised personnel make standard echocardiography time consuming and expensive. The same factors limit access to echocardiography and create delays in getting important results to the bedside. Furthermore, it is generally assumed that to perform any echocardiographic examination an examiner must be completely trained, certified, and examined. However, for answering simple questions, it may not be necessary to go to these extremes.

\section{PORTABLE ULTRASOUND MACHINES}

Recent advances in ultrasound technology have led to the development of fully portable ultrasound machines, which can provide immediate assessment of heart morphology and physiology at the time of the first examination of the patient. ${ }^{1}$ Now we can add sonography to the examination encounter. These personal imagers are appropriately named "ultrasonic stethoscopes" since they allow us to look into the chest and see the heart and its pathology during the physical examination. ${ }^{2}$ Visualising the heart with the ultrasound stethoscope as part of the physical examination provides additional information beyond what we can perceive with palpation and auscultation, and allows us to confirm rapidly a cardiac abnormality and often to make a specific diagnosis in any clinical setting. ${ }^{3}$ Direct visualisation of anatomy and function makes detection of preclinical pathology possible. The benefits include rapid evaluation of cardiac abnormalities (valve disease, cavity dilation, hypertrophy, pericardial effusion, wall motion abnormalities). The ultrasound extension of the physical examination can potentially identify these conditions, making earlier diagnosis and intervention possible. The routine physical cardiac examination can be extended by imaging and by obtaining limited quantitative measurements of the inferior vena cava (which provides an estimation of central venous pressure) and the abdominal aorta.

As this new approach evolves, it also raises the question of the clinical value of these personal imagers, in particular two major issues: what is the diagnostic ability of this modality compared to standard echocardiography, and what is its clinical utility in different clinical settings? The studies published on these topics are summarised in table $1^{4-10}$ and the results indicate that:

- personal ultrasound imagers improve the detection of important cardiovascular pathology compared with physical examination

- image quality of personal ultrasound imagers is adequate for performing a focused assessment of a limited number of two dimensional and Doppler parameters for the evaluation of cardiac anatomy and function

- these portable devices could become part of the clinical examination in selected patient groups, as a screening tool.

\section{USE IN CONSULTATION ROUNDS}

The study by Vourvouri and colleagues appearing in this issue of Heart adds further to the concept that these small portable ultrasound devices can be effectively applied as an ultrasonic stethoscope, with the goal of improving information obtained at physical examination. ${ }^{10}$ In this article, the authors investigated the impact of a small personal ultrasound imager during consultation rounds in patients referred for cardiac evaluation from non-cardiac departments. The results obtained using standard echocardiographic equipment were used for performance comparison and verification. The study found that the ultrasonic stethoscope provided sufficient information to the cardiologist in almost four out of five patients seen during consultation rounds. In one out of five patients, a further detailed examination was considered necessary, despite the echocardiographic examination with the ultrasound stethoscope, and in the vast majority of them a standard echocardiogram with Doppler study was required for the evaluation of the severity of regurgitant or 
Table 1 Published studies on the use of portable ultrasound machines

\begin{tabular}{|c|c|c|c|c|c|}
\hline Author & Aims & Patients & User & Results & \\
\hline Bruce $^{4}$ & $\begin{array}{l}\text { Screening of AAA } \\
\text { (SE as reference) }\end{array}$ & $\begin{array}{l}\text { Patients with HTN } \\
(n=125)\end{array}$ & Sonographer & $\begin{array}{l}\text { Sens } \\
\text { Spec } \\
\text { PPV } \\
\text { NPV }\end{array}$ & $\begin{array}{l}91 \% \\
96 \% \\
71 \% \\
99 \%\end{array}$ \\
\hline Spencer ${ }^{5}$ & $\begin{array}{l}\text { Diagnostic ability } \\
\text { ( } v \text { physical exam, } \\
\text { SE as reference) }\end{array}$ & $\begin{array}{l}\text { Cardiology clinic } \\
\text { ( } n=36,79 \mathrm{CV} \text { findings) }\end{array}$ & $\begin{array}{l}\text { BC cardiologist level II } \\
\text { SE by sonographer }\end{array}$ & Missed findings: & $\begin{array}{l}\text { PE } 59 \% \text {, } \\
\text { US } 29 \%\end{array}$ \\
\hline Goodkin $^{6}$ & $\begin{array}{l}\text { Diagnostic ability } \\
\text { (SE as reference) }\end{array}$ & $\begin{array}{l}\text { Critically ill patients } \\
(n=80 \text {, questions }=99)\end{array}$ & Experienced sonographer & Answer in $72 / 99$ (72\%) & \\
\hline Rugolotto ${ }^{7}$ & $\begin{array}{l}\text { Image quality } \\
\text { Accuracy } \\
\text { (SE as reference) }\end{array}$ & $\begin{array}{l}\text { Patients referred for SE } \\
(n=121)\end{array}$ & BC cardiologist level II & $\begin{array}{l}\text { Image quality: } \\
\text { 2-D data: } \\
\text { LVEDD: } \\
\text { Colour Doppler: }\end{array}$ & $\begin{array}{l}\text { NS } \\
\text { overall }<0.05 \\
\text { major NS } \\
p=0.04 \\
\text { overall } p<0.007 \\
\text { major NS }\end{array}$ \\
\hline Vourvouri ${ }^{8}$ & $\begin{array}{l}\text { Diagnostic accuracy } \\
\text { (SE as reference) }\end{array}$ & $\begin{array}{l}\text { Outpatients } \\
(n=114)\end{array}$ & Experience in echo & $\begin{array}{l}\text { LA size } \\
\text { LV size } \\
\text { LVEF } \\
\text { RWMA }\end{array}$ & $\begin{array}{l}\kappa=0.974 \\
\kappa=0.969 \\
93 \% \\
90 \%\end{array}$ \\
\hline Vourvouri9 & $\begin{array}{l}\text { Screening of LVH } \\
\text { (SE as reference) }\end{array}$ & $\begin{array}{l}\text { Outpatients with HTN } \\
(n=100)\end{array}$ & Experience in echo & $\begin{array}{l}\text { Agreement } \\
\text { Sens } \\
\text { Spec } \\
\text { PPV } \\
\text { NPV } \\
\text { Acc }\end{array}$ & $\begin{array}{l}93 \%(\kappa=0.77) \\
83 \% \\
95 \% \\
79 \% \\
96 \% \\
93 \%\end{array}$ \\
\hline Vourvouri ${ }^{10}$ & $\begin{array}{l}\text { Clinical utility } \\
\text { Cost effectiveness } \\
\text { (SE as reference) }\end{array}$ & $\begin{array}{l}\text { Patients from non-cardiac } \\
\text { departments } \\
\text { ( } n=107 ; 85 \% \text { pre-op) }\end{array}$ & Experience in echo & $\begin{array}{l}\text { Agreement } \\
\text { Sens } \\
\text { Spec } \\
\text { PPV } \\
\text { NPV } \\
\text { Cost reduction }\end{array}$ & $\begin{array}{l}96 \% \\
96 \% \\
96 \% \\
96 \% \\
96 \% \\
33.4 \%\end{array}$ \\
\hline
\end{tabular}

AAA, abdominal aortic aneurysm; BC, board certified; CV, cardiovascular; LA, left atrium; HTN, hypertension; LV, left ventricle; LVEDD, left ventricular end diastolic dimension; LVEF, left ventricular ejection fraction; LVH, left ventricular hypertrophy; NPV, negative predictive value; NS, not significant; PE, physical examination; PPV, positive predictive value; RWMA, regional wall motion abnormalities; SE, standard echocardiogram; US, ultrasound stethoscope.

stenotic lesions. With implementation of spectral Doppler and colour Doppler in the new generation of personal ultrasound imagers, a further reduction in the need for standard echocardiography can be anticipated. In addition, prevention of the extensive use of standard echocardiography by using the ultrasound stethoscope resulted in a cost reduction of $33 \%$. Based on these results, the authors conclude that during consultation rounds the ultrasonic stethoscope can help to make an instant diagnosis at the bedside, leading to a shortening of the time to diagnosis with equal efficacy to that achievable by standard echocardiography, but at a lower cost. However, the sensitivity of these devices for identifying certain conditions is still to be defined and the competence and training level of the examiner is an important aspect to consider.

All the published studies indicate that the greatest clinical utility of personal ultrasound imagers is when they are applied at the point-of-care of patients, providing immediate feedback. Who will then use these devices? Probably, their use will gradually evolve from the cardiologist to the internistcardiologist, general internist, primary care physician, registered nurse, medical school student, and allied health personnel. However, since incorrect information can be more harmful than no information, it requires those who want to use the ultrasound stethoscope to be properly trained to do so. Results from the only study aimed to prepare inexperienced examiners to evaluate common clinical disorders usually referred for echocardiographic evaluation indicate that medical residents with as little as three hours of training can use point-of-care echocardiography to improve substantially their assessment of left ventricular dysfunction and pericardial effusion. ${ }^{11}$ Whether additional training will improve their ability to assess valvar diseases will require further investigation. Thus, these portable systems may increase the availability of non-invasive imaging provided that trained staff are available. With this aim, the American Society of Echocardiography calls for "user-specific" training in the use of personal imagers for cardiac assessment. ${ }^{12}$ Those responsible for the use of these devices (cardiologists, sonographers, emergency room physicians) must be clearly defined and have adequate training. Protocols should specify appropriate indications for use and circumstances where referral of the patient for follow up with standard echocardiography is required.

\section{A NEW ERA}

We are entering a new and exciting era where the traditional approach to the clinical examination is likely to change with the availability of miniaturised ultrasound instruments that easily expand the information gained by our conventional senses. This field will continue to evolve as technology improves and devices with Doppler facilities become available. However, at present, we must be cautious about their widespread use and vigilant about the standards required of those who choose to use this technology, since the implications of missed or incorrect diagnoses resulting from technical deficiencies of the devices or to the operator's lack of training and experience are not known. Finally, further studies are clearly warranted to determine the impact of the ultrasound stethoscope on overall costs, patient management, and outcomes. 
Authors' affiliations

A Salustri, P Trambaiolo, Cardiology Unit, POI Portuense, Roma, Italy

\section{REFERENCES}

1 Roelandt JRTC. A personal ultrasound imager (ultrasound stethoscope) A revolution in the physical diagnosis! Eur Heart J 2002;23:523-27.

2 Salustri A, Trambaiolo P. Point-of-care echocardiography: small, smart and quick. Eur Heart J 2002;23: 1484-7.

3 Pandian NG. Ultrasound stethoscopy: adding another sense to the art and science of physical examination. Thoraxcentre J 2001:4:91-2.

4 Bruce CJ, Spittell PC, Montgomery SC, et al. Personal ultrasound imager: abdominal aortic aneurysm screening. J Am Soc Echocardiogr 2000;13:674-9.

5 Spencer KT, Anderson AS, Bhargava A, et al. Physician-performed point-of-care echocardiography using a laptop platform compared with physical examination in the cardiovascular patients. J Am Coll Cardiol 2001;37:2013-8.

6 Goodkin GM, Spevack DM, Tunick PA, et al. How useful is hand-carried bedside echocardiography in critically ill patients? J Am Coll Cardiol 2001;37:2019-22.
7 Rugolotto M, Hu BS, Liang DH, et al. Rapid assessment of cardiac anatomy and function with a new hand-carried ultrasound device $\left(O p t i \mathrm{O}^{T M}\right)$ : a comparison with standard echocardiography. Eur $J$ Echocardiogr 2001:4:262-9.

8 Vourvouri EC, Poldermans D, De Sutter J, et al. Experience with an ultrasound stethoscope. J Am Soc Echocardiogr 2002;15:80-5.

9 Vourvouri EC, Poldermans D, Schinkel AFL, et al. Left ventricular hypertrophy screening using a hand-held ultrasound device. Eur Heart $J$ 2002;23:1516-21

10 Vourvouri EC, Koroleva LY, Ten Cate FJ, et al. Clinical utility and cost effectiveness of a personal ultrasound imager for cardiac evaluation during consultation rounds in patients with suspected cardiac disease. Heart 2003;89:727-30

11 Alexander JH, Peterson ED, Chen AY, et al. Training and accuracy of non-cardiologists in simple use of point-of-care echo: a preliminary report from the Duke limited echo assessment project (LEAP). Thoraxcentre J 2001;4:105-10.

12 Seward JB, Douglas PS, Erbel R, et al. Hand-carried cardiac ultrasound (HCU) device. A report from the echocardiography task force on new technology of the nomenclature and standard committee of the American Society of Echocardiography. J Am Soc Echocardiogr 2002;15:369-73.

\section{IMAGES IN CARDIOLOGY}

\section{The "pop phenomenon" detected by phased array intracardiac echocardiography}

R adiofrequency ablation with an irrigation catheter is available to ablate ventricular tachycardia in cases with a structural heart disease. The "pop phenomenon" associated with the use of this system remains crucial but direct visualisation of the "pop phenomenon" has not been reported. A deflectable $8.5 \mathrm{MHz}$ phased array intracardiac echocardiography (ICE) catheter (Acu Nav, Acuson Inc) presented in a canine model the visual aspect of a real time "pop phenomenon" on the apical posterior wall of the left ventricle in vivo. Using a temperature controlled system, low power energy was delivered to the left ventricle for 45 seconds. The mean temperature was $55^{\circ} \mathrm{C}$ with a mean power of $25 \mathrm{~W}$. Suddenly, the ablated lesions were vividly demonstrated by the presence of a notable increase in tissue density and the central hypoechoic area (below left). Simultaneously, we found a bubble formation in the left ventricle. The real time measurements of the maximum width and depth of the hyperechoic lesion and the central hypoechoic area were 13.8 and $9.0 \mathrm{~mm}$, and 6.1 and $4.5 \mathrm{~mm}$, respectively. A histological examination revealed sharply demarcated ablated lesions and a central

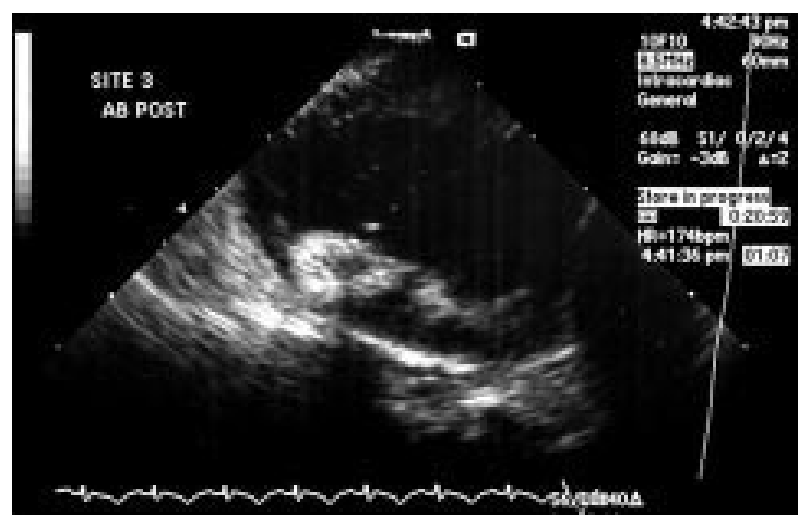

crater formation (below right). Macroscopically, the maximum width and depth of the ablated lesion and the central crater were 13.2 and $8.8 \mathrm{~mm}$, and 6.2 and $4.7 \mathrm{~mm}$, respectively. The sizes of the ablated lesion and the crater on ICE corresponded to those of the macroscopic measurements.

Phased array ICE may be useful in the direct identification of an ablated lesion and crater formation when a "pop phenomenon" occurs during low power ablation.

M Takagi
I Toda
J Yoshikawa
m7424580@msic.med.osaka-cu.ac.jp

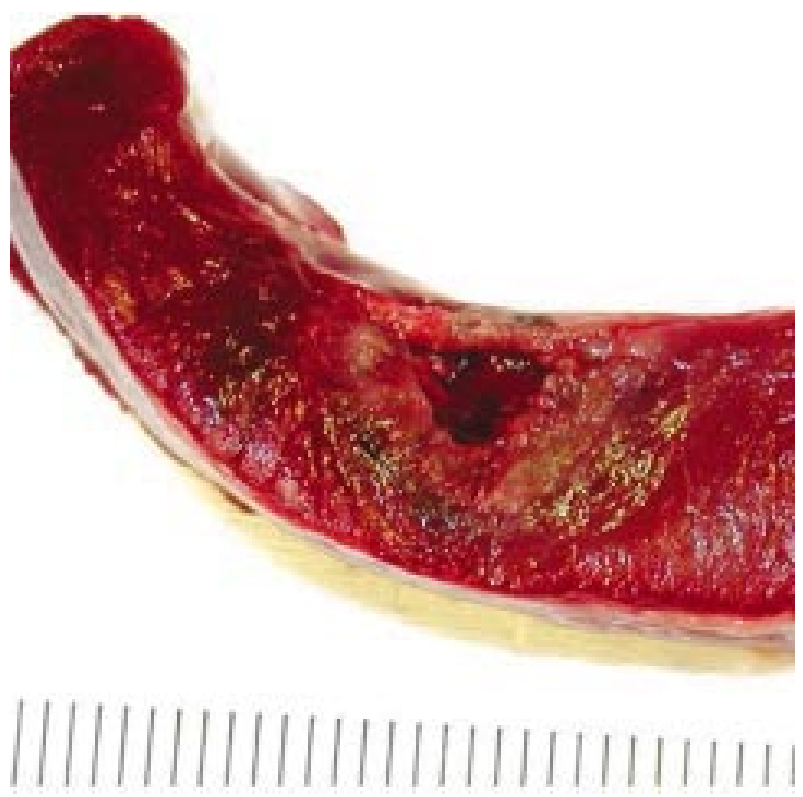

